\title{
Data Framework and Related Technologies of Electronic Information System
}

\author{
Hua Wang, Xianyu Li, Jianqun Zheng, Zhengmao Chen and Peitao Liu \\ Beijing Shi QingHe DaLou Zi Jiu, Beijing, 100085, China \\ email: kking127@sohu.com
}

Keywords: Data Framework; Data Processing Technology; Electronic Information System

\begin{abstract}
Based upon the synopsis of big data and electronic information system, this paper puts up the data framework, carries out practical analysis and systematic application of data collecting technology, data synchronization technology, data excavation technology, and data analysis technology, and describes the analysis model of system data flow. This will benefit the dynamic improvement of electronic information system.
\end{abstract}

\section{Introduction}

Electronic information system is developed to organize related resources, conduct information processing and actualize business activities. Along with the theoretical and practical development of big data and related technologies, electronic information system needs must be properly improved to satisfy the requirements of dynamic function evolvement and swift mission realization.

Big data is information assets of magnanimity, diversification and high increase rate that possess strong capabilities of decision-making sustainment, percipience discovery and flow optimization. It is marked by Volume, Variety, Velocity and Value. Big data is essentially systematic and intellectualized information organization models and service modes that more meet the needs of business information and decision activities [1][2][3].

To view from the life cycle in information system, big data need to pass through the nodes of data provision, storage management, calculation disposing, data analysis, and knowledge exhibition. Big data technologies are a series of technologies that deal with a great deal of structured, semi-structured and non-structured data by nontraditional tools, and comprise data analysis technology, calculation technology and storage management technology.

Big data manages magnanimity data by new methods, and constitutes one system of independent operation and flexible availability. It can strengthen data circulation degree and provides users with celerity approach to the obtainment of business dynamic and state information. Big data aims at key demands of business decision activities, deals with complicated data by new means and tools, intellectually syncretizes and analyses present and history data, and provides precise trend prognostication and conclusion report, so that use unit can finely grasp business pulses and dominate activity courses.

\section{Data Framework}

Data framework of electronic information system is mainly composed of support type, service type, computing type, storage type and security type [3][4]. It is shown in Figure 1. 


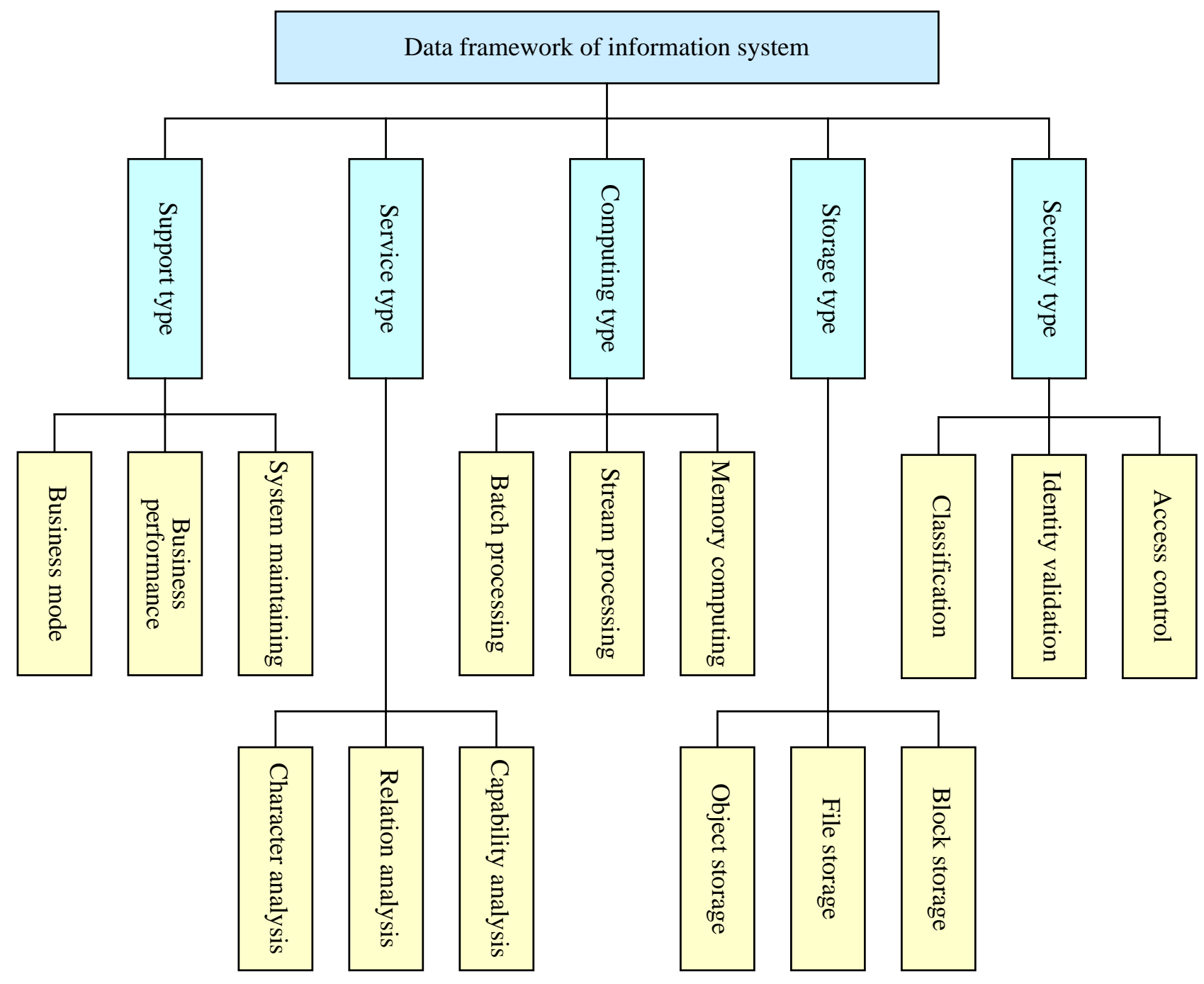

Fig. 1. Data framework of electronic information system

(1) Support type contributes to application implementation, and comprises the related aspects of business mode, business performance and system maintaining.

(2) Service type contributes to application analysis, and comprises the aspects of character analysis, relation analysis and capability analysis.

(3) Computing type contributes to computation mode, and comprises the aspects of batch processing, stream processing and memory computing.

(4) Storage type contributes to platform basis, and comprises the aspects of object storage, file storage and block storage.

(5) Security type contributes to application protection, and comprises the aspects of classification, identity validation and access control.

We should investigate algorithms of exactness apperception and effect obtainment of multi-source and different fabrication data, set up real-time and intellectual framework of data collecting, so as to accomplish effective processing of non-structural data. In the meantime, data synchronization technology, data excavation technology, and data analysis technology are researched to support the construction and operation of data system.

\section{Data Synchronization Technology}

Data system need currently satisfy demands for diverse applications, effectively link up with various information systems, and duly updated to reflect data change, so as to ensure consistence, integrality and veracity of data system over transverse and lengthwise departments [5][6].

Message bus model of special data is devised to achieve system data synchronization by means 
of message registration, message distribution and data processing services. Message bus model of special data is shown in Figure 2.

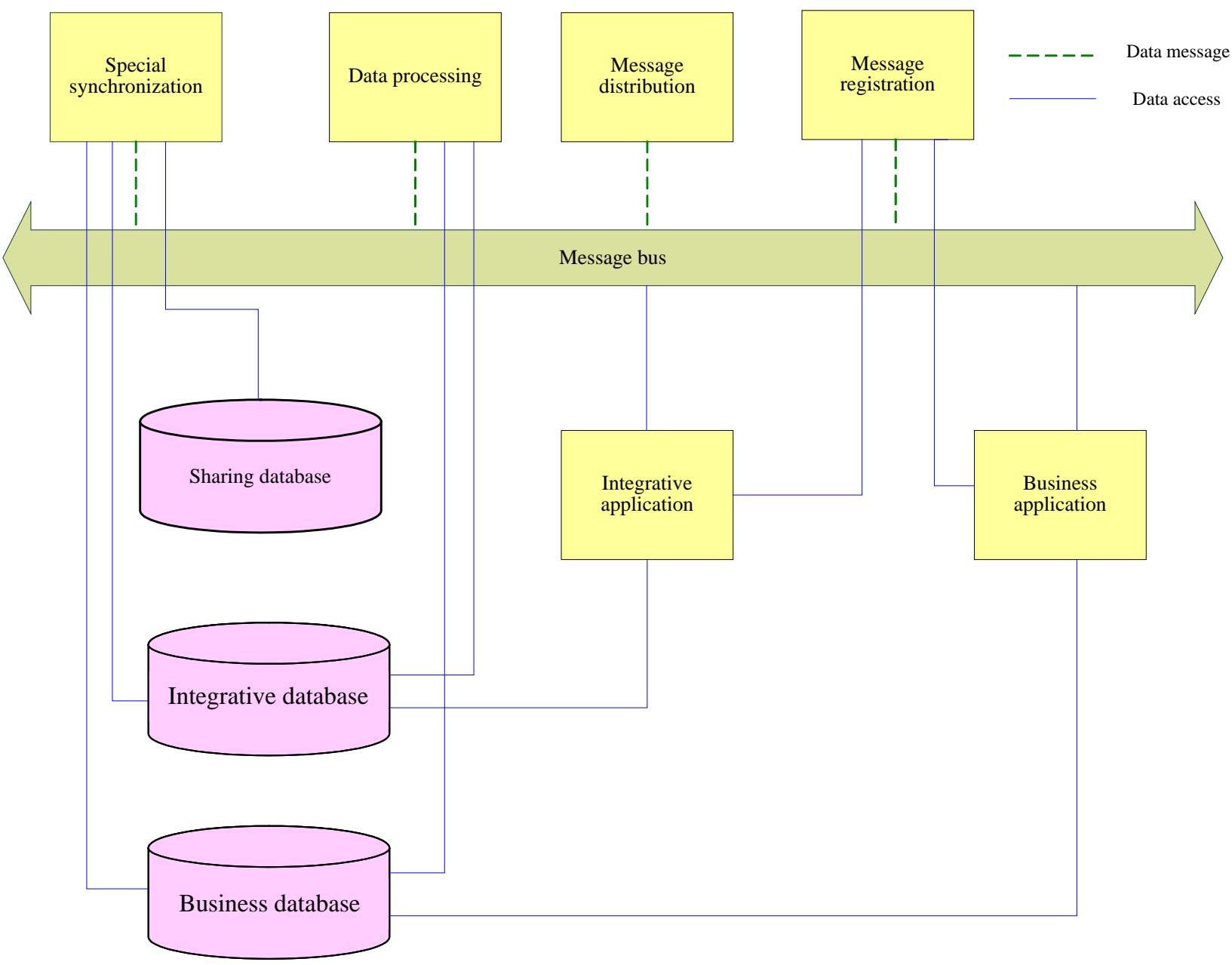

Fig. 2. Message bus model of special data

(1) Sponsor registers message type and data renewal description by message registration service.

(2) Accepter registers renewal business message.

(3) Sponsor registers updates business database and send out renewal message.

(4) Message registration service sends out message to all the accepters of data processing service via message bus.

(5) Data processing service updates the related database in light of renewal queue.

(6) Accepter receives renewal message and achieve data inquiry and transaction.

(7) Special synchronization service is used to synchronize superior and subordinate information.

In addition, the model of difference synchronization is brought forward to achieve the real time synchronization and updating in wide field, and related hiberarchy is shown in Figure 3.

(1) Physics link layer contributes to the application basis and dynamic extension of information system, and comprises a series of communication means.

(2) Transport protocol layer contributes to the data sharing, dynamic adjustment and mutual manipulation, and comprises various communication protocols.

(3) Synchronization transfer service layer contributes to the application oriented construction of information system, and comprises address mapping and transfers transmission services. Address mapping service sustains TCP/IP address and other protocol address conversion/transition; transfers service supports the transfer of related transport protocols.

(4) Synchronization transaction service layer contributes to particular application adjustment of information system, and comprises various synchronization services besides transfers, such as 
difference comparison, database read-write access, message processing and coding management.
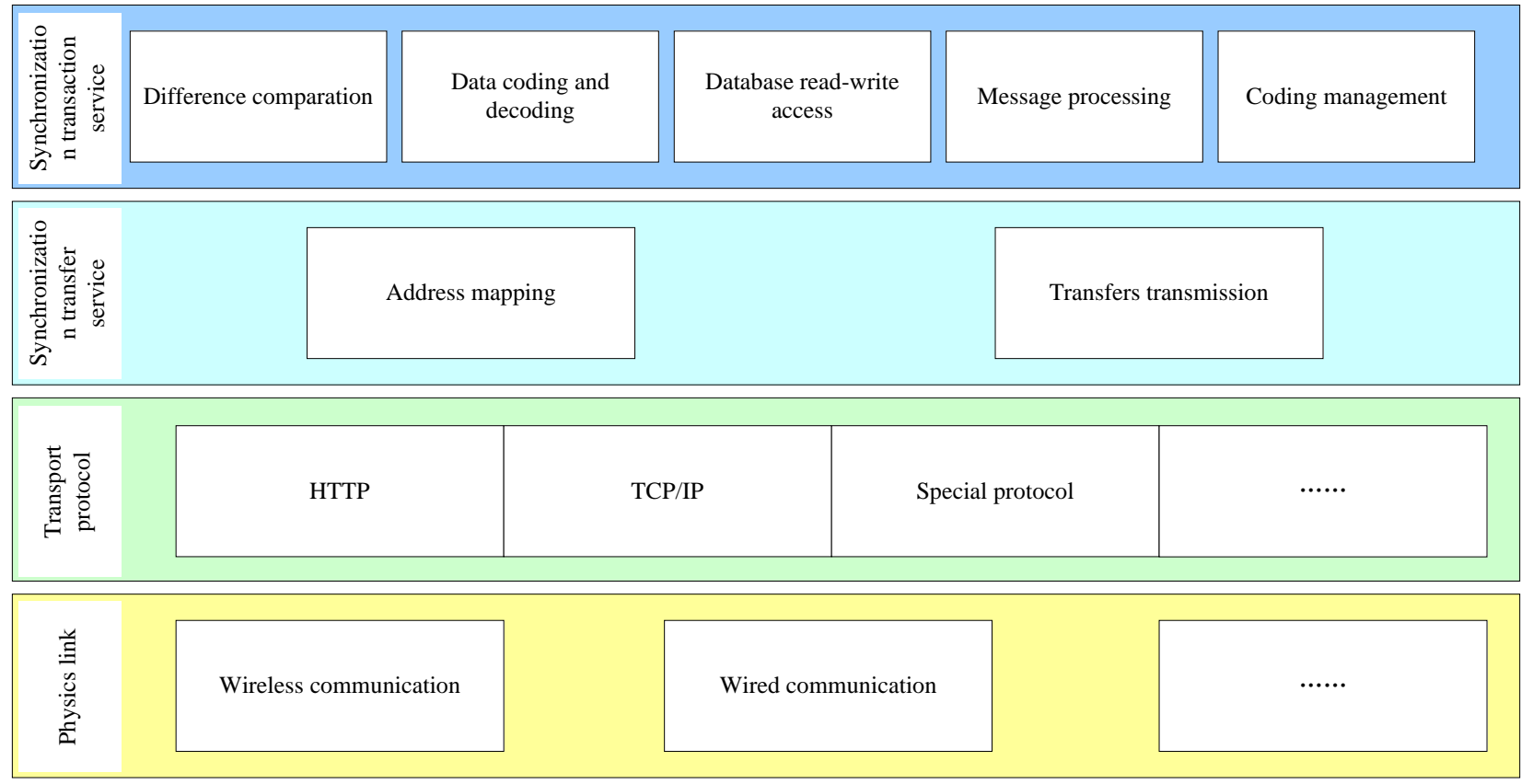

Fig. 3. The model of difference synchronization

The system adopts unilateralism data synchronization, and system data is delivered from source end while synchronizing. Firstly we capture discrepancy data by means of time stamp and main text, and keep a record of these data; secondly the discrepancy data could be obtained by data access service and transferred to object server by general transfer service; and lastly asynchronism database access is carried out at object server so as to achieve data synchronization.

\section{Data excavation Technology}

Data excavation can discover connotative information of deep arrangement, assistant institution and effective decision-making, and propel information dominance into decision dominance. Data excavation comprises associated rules excavation, classification and forecast, clustering analysis and isolated point analysis [5][6].

Rule excavation is to discover frequency mode, cause and effect framework and relevancy among databases; classifying and forecast is to find out models or functions that describe and differentiate data classes or concepts, and forecast unknown type labels of objects; clustering analysis is to generate type labels in the light of maximum object comparability within one type and minimum object comparability among types; isolated point analysis is to investigate data models or behavior principles of isolated points and related special applications. Of course, there are diverse algorithms and methods for the above technologies.

\section{Data Analysis Technology}

During the process of data system construction, we especially reflect present information field technologies, take data application characteristics into consideration, and set up the model of system data flow that includes data customization and acquirement, filtration and conversion, evolvement and analysis, and syncretization and exhibition [6][7]. The analysis model of system data flow is shown in Figure 4. 


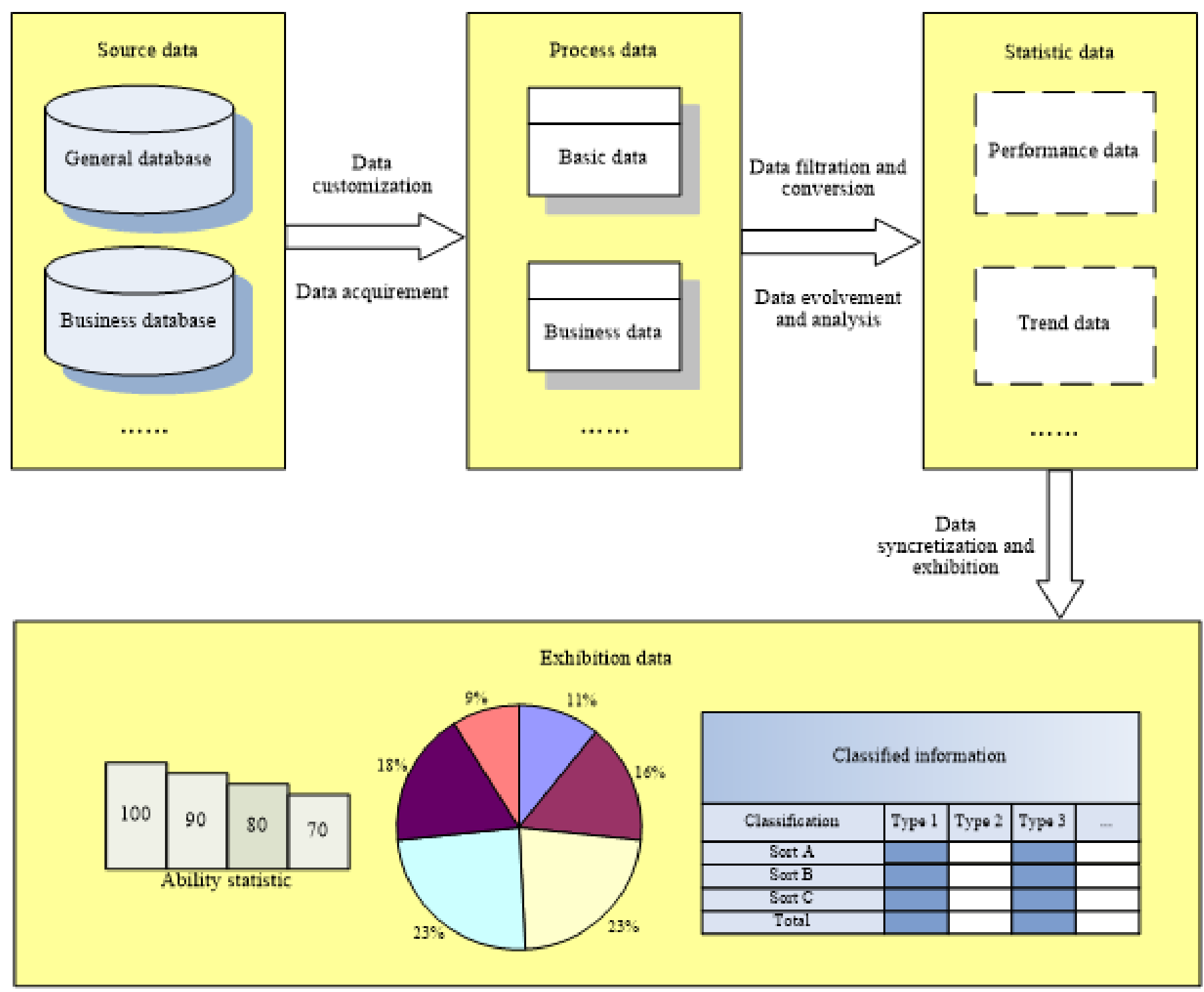

Fig. 4. The analysis model of system data flow

(1) Data customization and acquirement is mostly to aim at related element databases and dispersive data of various sources, fully comprehend data definition, and establish proper and exercisable databases and sources, define extractive data dimensionalities, and then extract needful data from databases and write them into process slot.

(2) Data filtration and conversion is to regulate related cleaning manipulation and eliminate doubtful data notes, so as to avoid data variousness, repetition and fragment; it is helpful to the further analysis of business data and belongs to typical process of pattern distinguishment.

(3) Data evolvement and analysis is to deduce future value from history data by the exponential model of thrice smoothness, and the forecast outcome is automatically presented if consumer chooses one intending time where the weight number is repeatedly selected so as to minimize the forecast error.

(4) Data syncretization and exhibition is to explore and describe related laws and tracks of system data. Some latent and important information can be unearthed by data disposing, i.e. statistic chart or mathematics model, and the evolution and trend of business indexes and related objects are concisely depicted and forecasted.

\section{Conclusion}

During the development of electronic information system, data framework should be established, and the related technologies such as data collecting technology, data synchronization technology, data excavation technology, and data analysis technology are applied to the construction and 
operation of data system. Moreover, we should further advance the improvement of system architecture and data requirement, increase the data standardization and mutual operation of information system, so that information system can smoothly meet changing need and achieve desired objective.

\section{Acknowledgement}

We are grateful to the specialists and secretaries for their insight and guidance, understanding and patience, and they are a constant source of support and encouragement.

\section{References}

[1] Tu Zipei. Big Data [M]. Guangxi Teachers College Press, 2012.

[2] Wang Y Z, Jin X L, Cheng X Q. Network big data: Present and future [J]. Chinese Journal of Computers, 2013 (36) 1-15.

[3] Li GuoJie. The Scientific Value of Big Data [J]. Communicatiom of CCF, 2012 (9): 8-15.

[4] Gao Wei. The Fundamental Computing Platform for Application of Big Data [J]. Information Technology and Standardization, 2013 (12) 20-23.

[5] Meng Xiaofeng, Ci Xiang. Big Data Management: Concepts, Techniques and Challenges [J]. Journal of Computer Research and Development, 2013 (1) 146-169.

[6] Gong Xueqing, Jin Cheqing, Wang Xiaoling, Zhang Rong, Zhou Aoying. Data-intensive science and engineering: Requirements and challenges [J]. Chinese Journal of Computers, 2012 35(8) 1563-1578.

[7] Fan Xianxue, Jin Xinghua. Communication network structure design for data center [J]. Command Information System and Technology, 2014 (3) 64-69. 